\title{
Evaluating Mechanical Ventilators Using Multi Criteria Decision Making Techniques
}

\author{
https://doi.org/10.3991/ijoe.v17i07.21769 \\ Qasem Alyazji ${ }^{(凶)}$, Gulsum Asiksoy \\ Near East University, Nicosia / TRNC, Turkey \\ K yazgy@hotmail.com
}

\begin{abstract}
Mechanical ventilator (MV) is used to help the patient breathe by delivering gas to the lungs at a certain rate using positive pressure. The complex evaluation of mechanical ventilator devices at present time is a very important and topical issue, due to the presence of many mechanical ventilator companies, as it seems that the process of evaluation and selection of ventilator equipment needs strong experience in this field. Our paper show that multi criteria decision making (MCDM) methods can be applied to comparing and evaluating some alternatives of mechanical ventilator devices. This study will determine new methodology to help the decision makers to choosing the best mechanical ventilator among the five alternatives based on eight criteria; Cost of the MV device; Maximum Inspiratory flow; Maximum Pressure; Tidal volume; PEEP; Weight of ventilator; Screen size and Internal battery time. This study used two techniques; TOPSIS technique and PROMETHEE II technique. Our paper used the same weights criteria in these two techniques. The weight for each criteria should be determined by the medical engineer expert and the decision makers. Choosing mechanical ventilator will affect the quality of the therapeutic and diagnostic processes, the way the treating doctor works, and also affect the patient's comfort. Because of these reasons, we designed a new methodology based on MCDM. The aim of this research is to design a new method for evaluating ventilator machines and medical technology for the purpose of purchasing ventilator devices in hospitals. The choice of the appropriate ventilator device is a big problem among the decision-makers so that its purchase must be transparent. The new method will serve as the basis for making the decision in purchasing a ventilator. This research dealing with effective procurement will become part of the entire decision-making chain as the expected usability of the device also must be considered.
\end{abstract}

Keywords-Mechanical ventilator, multiple-criteria decision making, TOPSIS, PROMETHEE

\section{$1 \quad$ Introduction}

A mechanical ventilator helps the patient to breathe only and does not treat the illness, so the patient's basic condition must be determined and treated, as the patient's stay on the ventilator for a long period leads to harm to the patient [1]. The ventilator is not a treatment for a disease, but it is used during treatment to stabilize the patient's condition, for example in the case of patients with Coronavirus, the ventilator does not 
treat the virus, but it helps the patient to breathe to survive until the body recovers from the virus and defeats it, or when pharmacological treatments work. Mechanical ventilation settings that are not appropriate for the patient's condition may lead to a deterioration of the condition patient and can cause more damage [2]. On the other hand, the settings of the mechanical ventilator are below the optimum level can lead to an increase in the patient's stay in the ICU and thus increase the patient's treatment cost. Using a mechanical ventilator for a long time may lead to problems such as pneumonia [3]. Airway pressure and tidal volume should be set correctly in mechanical ventilators; applied high pressure may cause damage to healthy lung units. The ventilator must be applied for a short period of time, to avoid complications arising from the use of a ventilator [4].

When connecting a ventilator to a patient, there are some major parameters that must be setting to the ventilator, such as; The volume of air that enters the lung during each breathing cycle which is called tidal volume $\left(V_{t}\right)$, Number of breaths per minute which is called Respiratory rate (RR), volume of air expired per minute which is called Minute ventilation (VE), the amount of oxygen delivered to the patient which is called Fraction of inspired oxygen (FIO2), the inspiratory time compared to the expiratory time which is called I:E Ratio, the speed which volume is delivered which is called Flow rate, the value of trans respiratory system pressure at end expiration which is called PEEP (Positive End Expiratory Pressure) ; and the Maximum pressure applied to the patient's proximal airway which is called Peak Inspiratory Pressure (PIP) [5]. The previously mentioned variables can decide the patient's full breathing cycle, and the primary objective of the mechanical ventilator is to facilitate gas exchange, without adding a lot of pressure that may cause harm to a healthy lung.

The aim of this research is to design new method for evaluating ventilator machines and medical technology for the purpose of purchasing ventilator devices in hospitals. The choice of the appropriate ventilator device is in a big problem among the decisionmakers so that its purchase must be transparent. The new method will serve as the basis for making the decision in purchasing a ventilator. We will evaluate the ventilator device in terms of technical and clinical aspects, also in terms of cost and ease of use. This research dealing with effective procurement will become part of the entire decisionmaking chain as the expected usability of the device also must be considered. At the present time, the purchases of medical technology in most countries of the world lack any coordination of the methods of decision-making in the purchase of a ventilator. This study only addresses the issues of choosing a suitable ventilator. The main difficulties in any evaluation of technology are solving the problem of evaluating the effective component of devices. The devices may not directly affect the criteria related to the quality of life, as they affect the quality of the diagnostic and therapeutic processes, the way the treating physician works and fundamentally affect the patient's comfort. To this end, we have used multi criteria decision-making (MCDM) methods to choose the best ventilator. This study aims to assist medical engineers, doctors and decisionmakers in making the appropriate decision to choose the best ventilator. Choosing the best device depends on several important criteria such as cost of the MV device; maximum inspiratory flow; maximum pressure; tidal volume; PEEP; weight of ventilator; screen size and internal battery time. 


\section{Methods}

Multi criteria decision making (MCDM) methods applied to help decision makers for evaluate alternative mechanical ventilator by using TOPSIS technique \& PROMETHEE II technique. TOPSIS technique \& PROMETHEE II technique are very important kind of MCDM methods in order to comparing and evaluating some of alternatives which is based on multiple criteria and is inconsistent. TOPSIS technique which is the full name "Technique for Order Preference by Similarity to an Ideal Solution". TOPSIS technique was first introduced by Yoon \& Hwang (1981) [6]. PROMETHEE II technique which is the full name "preference ranking organization method for enrichment evaluation". TOPSIS and PROMETHEE II techniques are used in many different areas, especially medical, also applied to a wide range of engineering and medical decision-making problems that contain conflicting criteria.

The basic processes for the TOPSIS technique and PROMETHEE II technique are displayed below.

\subsection{TOPSIS technique}

TOPSIS technique depend on the calculating the best ideal solution and ideal worst solution. During this method, we should be classify each criteria; benefit criteria or nonbenefit criteria. The maximum value of non-benefit criteria is called the ideal worst solution; also, the minimum value of benefit criteria is called ideal worst solution. The maximum value of the benefit criteria is called ideal best solution; also, the minimum value of non-benefit criteria is called ideal best solution [7]. The TOPSIS is easy to use, not requiring minimization of variables, and applicable in many different areas [8]. TOPSIS is based on the simple process; it is programmable and easy to apply. The TOPSIS method is easy in terms of maintaining the same amount of steps regardless of problem size or number of alternatives [7, 8]. The following are the steps to be performed in TOPSIS technique [9]

Step 1: The value for weight criteria $\left(W_{j}\right)$ and weightage of each criteria must be should be defined as

$$
\sum_{j=1}^{n} W_{j}=1
$$

where $\mathrm{n}$ is the number of criteria

Step 2: Determine the matrix of normalized value. The normalized value $N_{i j}$ must be calculated by the following equation:

$$
N_{i j}=\frac{x_{i j}}{\sqrt{\sum_{i=1}^{z} x_{i j}^{2}}}
$$

Where $\mathrm{x}_{\mathrm{ij}}$ the value of the $\mathrm{i}^{\text {th }}$ alternative and $\mathrm{j}^{\text {th }}$ attribute/ criteria.

Step 3: Multiply the normalize value with the weight of each criteria as the equation number (3); and this called $\left(A_{i j}\right)$.

$$
A_{i j}=N_{i j} \mathrm{X} W_{j}
$$


Step 4: Ideal best solution $\left(V^{+}\right)$and ideal worst solution $\left(V^{-}\right)$must be calculated by applying the following equation (4 and 5), respectively.

$$
\begin{aligned}
& \mathrm{B}^{+}=\left\{\left(\max _{\mathrm{i}} \mathrm{A}_{\mathrm{ij}} \backslash \mathrm{j} \in \mathrm{C}_{\mathrm{b}}\right),\left(\min _{\mathrm{i}} \mathrm{A}_{\mathrm{ij}} \backslash \mathrm{j} \in \mathrm{C}_{\mathrm{c}}\right)\right\}=\left\{\mathrm{A}_{\mathrm{j}}^{+} \backslash \mathrm{j}=1,2, \ldots, \mathrm{m}\right\} \\
& \mathrm{B}^{-}=\left\{\left(\min _{\mathrm{i}} \mathrm{A}_{\mathrm{ij}} \backslash \mathrm{j} \in \mathrm{C}_{\mathrm{b}}\right),\left(\max _{\mathrm{i}} \mathrm{A}_{\mathrm{ij}} \backslash \mathrm{j} \in \mathrm{C}_{\mathrm{c}}\right)\right\}=\left\{\mathrm{A}_{\mathrm{j}}^{-} \backslash \mathrm{j}=1,2, \ldots, \mathrm{n}\right\}
\end{aligned}
$$

Where $\mathrm{C}_{\mathrm{b}}$ is benefit criteria and $\mathrm{C}_{\mathrm{c}}$ is non-benefit criteria, for $\mathrm{i}=1,2,3 \ldots \mathrm{m} ; \mathrm{j}=1,2,3$ $\ldots \mathrm{n}$ : Best ideal solution $\mathrm{B}^{+}$is the maximum value of the benefit criteria and the minimum value for the non-benefit criteria. Worst ideal solution $B^{-}$is the maximum value of the non-benefit criteria and the minimum value of the criteria.

Step 5: Compute the difference distance of each alternative from the best ideal solution and the difference distance of each alternative from the worst ideal solution, by using equation number $(6,7)$ respectively:

- Ideal Positive Distance $\left(D_{i}^{+}\right)$

$$
M_{i}^{+}=\sqrt{\sum_{j=1}^{m}\left(B_{i j}-B_{j}^{+}\right)^{2}}, j=1,2, \ldots, m
$$

- Ideal Negative Distance $\left(D_{i}^{-}\right)$

$$
M_{i}^{-}=\sqrt{\sum_{j=1}^{m}\left(B_{i j}-B_{j}^{-}\right)^{2}}, j=1,2,3 \ldots ., m
$$

Step 6: Performance core $\left(P_{i}\right)$ must be calculated using the equation number (8):

$$
P_{i}=\frac{M_{i}^{-}}{M_{i}^{+}+M_{i}^{-}}, \mathrm{i}=1,2,3 \ldots ., \mathrm{m}
$$

Where $0 \leq P_{i} \leq, \mathrm{i}=1,2,3 \ldots \mathrm{m}$

So, based on the performance score $\left(P_{i}\right)$, rank the alternative mechanical ventilator.

\section{Data Collection, Criteria and Application}

This study will determine new methodology to help the decision makers the choice of the best mechanical ventilator among the five alternatives; $A_{1}=$ Medtronic Puritan Bennett 980 [10]; $A_{2}=$ GE Carescape R860 [11]; $A_{3}=$ Hamilton G5/S1 [12]; $A_{4}=$ Dräger Evita V500 [13] and $A_{5}=$ Maquet SERVO-U [14]. Evaluate and compare between these alternative based on eight criteria; $C_{1}=$ cost of the MV device, have weightage $0.35 ; C_{2}=$ Maximum Inspiratory flow, have weightage $0.1 ; C_{3}=$ Maximum Pressure, have weightage $0.1 ; C_{4}=$ Tidal volume, have weightage $0.15 ; C_{5}=$ PEEP, have weightage $0.1 ; C_{6}=$ Weight of ventilator, have weightage $0.05 ; C_{7}=$ Screen size, have weightage 0.05 and $C_{8}=$ Internal battery time, have weightage 0.1 .

The weight of each criteria was determined based on the opinions of medical engineers, ICU doctors and the users. It is unusual to have the cheapest device to be the most comfortable and the safest for the patient. Evaluation and selection of a ventilator 
is a major concern for hospitals because of its great importance in assisting in the treatment of patients after major and medium surgical operation and also during the patient's stay in ICU. Therefore, it was necessary to design a methodology for the decision-making of mechanical ventilators, based on some technical and clinical criteria for ventilator devices.

The above TOPSIS Steps will be applied in the evaluation and selection of the mechanical ventilator. Data and information in Table 1 were collected with the help of medical engineers and intensive care doctors \& nurses, as well as with the help of manufacturer catalogs.

Table 1. Data collection for mechanical ventilator criteria

\begin{tabular}{|c|c|c|c|c|c|c|c|c|}
\hline & $\begin{array}{c}\boldsymbol{C}_{\mathbf{1}} \\
\mathbf{\$})\end{array}$ & $\begin{array}{c}\boldsymbol{C}_{\mathbf{2}} \\
(\mathbf{l} / \mathbf{m i n})\end{array}$ & $\begin{array}{c}\boldsymbol{C}_{\mathbf{3}} \\
(\mathbf{c m H 2 O})\end{array}$ & $\begin{array}{c}\boldsymbol{C}_{\mathbf{4}} \\
(\mathbf{m l})\end{array}$ & $\begin{array}{c}\boldsymbol{C}_{\mathbf{5}} \\
(\mathbf{c m H 2 O})\end{array}$ & $\begin{array}{c}\boldsymbol{C}_{\mathbf{6}} \\
(\mathbf{k g})\end{array}$ & $\begin{array}{c}\boldsymbol{C}_{\mathbf{7}} \\
(\mathbf{i n c h})\end{array}$ & $\begin{array}{c}\boldsymbol{C}_{\mathbf{8}} \\
(\mathbf{m i n})\end{array}$ \\
\hline $\boldsymbol{A}_{\mathbf{1}}$ & 30,000 & 150 & 100 & $2-2500$ & $0-45$ & 31 & 15 & 60 \\
\hline $\boldsymbol{A}_{\mathbf{2}}$ & 32,000 & 160 & 100 & $2-2000$ & $1-50$ & 31 & 15 & 30 \\
\hline $\boldsymbol{A}_{\mathbf{3}}$ & 27,000 & 180 & 100 & $2-2000$ & $0-50$ & 42 & 15 & 60 \\
\hline $\boldsymbol{A}_{\mathbf{4}}$ & 29,000 & 180 & 95 & $2-3000$ & $0-50$ & 23 & 17 & 30 \\
\hline $\boldsymbol{A}_{\mathbf{5}}$ & 33,000 & 200 & 120 & $2-4000$ & $1-50$ & 19 & 15 & 120 \\
\hline
\end{tabular}

Table 2. Calculate normalized performance value

\begin{tabular}{|c|c|c|c|c|c|c|c|c|}
\hline & 0.35 & 0.1 & 0.1 & 0.15 & 0.1 & 0.05 & 0.05 & 0.1 \\
\hline & $\begin{array}{l}C_{1} \\
(\$)\end{array}$ & $\begin{array}{c}C_{2} \\
(l / m i n) \\
\end{array}$ & $\begin{array}{c}C_{3} \\
(\mathrm{cmH} 2 O) \\
\end{array}$ & $\begin{array}{c}C_{4} \\
(m l) \\
\end{array}$ & $\begin{array}{c}C_{5} \\
(\mathrm{cmH} 2 O) \\
\end{array}$ & $\begin{array}{c}C_{6} \\
(\mathrm{~kg})\end{array}$ & $\begin{array}{c}C_{7} \\
\text { (inch) }\end{array}$ & $\begin{array}{c}C_{8} \\
(m i n)\end{array}$ \\
\hline$A_{1}$ & 0.4431 & 0.383 & 0.432 & 0.399 & 0.410 & 0.458 & 0.435 & 0.392 \\
\hline$A_{2}$ & 0.4726 & 0.409 & 0.432 & 0.319 & 0.455 & 0.458 & 0.435 & 0.196 \\
\hline$A_{3}$ & 0.3988 & 0.460 & 0.432 & 0.319 & 0.455 & 0.620 & 0.435 & 0.392 \\
\hline$A_{4}$ & 0.4283 & 0.460 & 0.410 & 0.478 & 0.455 & 0.340 & 0.493 & 0.196 \\
\hline$A_{5}$ & 0.4874 & 0.511 & 0.519 & 0.638 & 0.455 & 0.280 & 0.435 & 0.784 \\
\hline
\end{tabular}

Table 3. Next, we multiply the weight for each criteria with the normalized performance values of each cell, this is called Weighted Normalized Decision Matrix

\begin{tabular}{|c|c|c|c|c|c|c|c|c|}
\hline & $\mathbf{0 . 3 5}$ & $\mathbf{0 . 1}$ & $\mathbf{0 . 1}$ & $\mathbf{0 . 1 5}$ & $\mathbf{0 . 1}$ & $\mathbf{0 . 0 5}$ & $\mathbf{0 . 0 5}$ & $\mathbf{0 . 1}$ \\
\hline & $\begin{array}{c}\boldsymbol{C}_{\mathbf{1}} \\
(\$)\end{array}$ & $\begin{array}{c}\boldsymbol{C}_{\mathbf{2}} \\
(\boldsymbol{l} / \mathbf{m i n})\end{array}$ & $\begin{array}{c}\boldsymbol{C}_{\mathbf{3}} \\
(\mathbf{c m H} 2 \boldsymbol{O})\end{array}$ & $\begin{array}{c}\boldsymbol{C}_{\mathbf{4}} \\
(\mathbf{m l})\end{array}$ & $\begin{array}{c}\boldsymbol{C}_{\mathbf{5}} \\
(\mathbf{c m H} 2 \boldsymbol{O})\end{array}$ & $\begin{array}{c}\boldsymbol{C}_{\mathbf{6}} \\
(\boldsymbol{k g})\end{array}$ & $\begin{array}{c}\boldsymbol{C}_{\mathbf{7}} \\
(\text { inch })\end{array}$ & $\begin{array}{c}\boldsymbol{C}_{\mathbf{8}} \\
(\mathbf{m i n})\end{array}$ \\
\hline $\boldsymbol{A}_{\mathbf{1}}$ & 0.1550 & 0.0383 & 0,0432 & 0.0598 & 0.041 & 0.0229 & 0.0217 & 0.0392 \\
\hline $\boldsymbol{A}_{\mathbf{2}}$ & 0.1654 & 0.0409 & 0.0432 & 0.0478 & 0.0455 & 0.0229 & 0.0217 & 0.0196 \\
\hline $\boldsymbol{A}_{\mathbf{3}}$ & 0.1395 & 0.046 & 0.0432 & 0.0478 & 0.0455 & 0.031 & 0.0217 & 0.0392 \\
\hline $\boldsymbol{A}_{\mathbf{4}}$ & 0.1499 & 0.046 & 0.041 & 0.0717 & 0.0455 & 0.017 & 0.0246 & 0.0196 \\
\hline $\boldsymbol{A}_{\mathbf{5}}$ & 0.1706 & 0.051 & 0.0519 & 0.0957 & 0.0455 & 0.014 & 0.0217 & 0.0784 \\
\hline
\end{tabular}


Table 4. Determine ideal best solution and ideal worst solution

\begin{tabular}{|c|c|c|c|c|c|c|c|c|}
\hline & $\begin{array}{c}\text { non } \\
\text { benefit } \\
\text { Criteria }\end{array}$ & $\begin{array}{l}\text { benefit } \\
\text { Criteria }\end{array}$ & $\begin{array}{l}\text { benefit } \\
\text { Criteria }\end{array}$ & $\begin{array}{l}\text { benefit } \\
\text { Criteria }\end{array}$ & $\begin{array}{l}\text { benefit } \\
\text { Criteria }\end{array}$ & $\begin{array}{c}\text { non } \\
\text { benefit } \\
\text { Criteria }\end{array}$ & $\begin{array}{l}\text { benefit } \\
\text { Criteria }\end{array}$ & $\begin{array}{l}\text { benefit } \\
\text { Criteria }\end{array}$ \\
\hline & $\begin{array}{l}C_{1} \\
(\$)\end{array}$ & $\begin{array}{c}C_{2} \\
(l / m i n)\end{array}$ & $\begin{array}{c}C_{3} \\
(\mathrm{cmH} 2 \mathrm{O})\end{array}$ & $\begin{array}{c}C_{4} \\
(m l)\end{array}$ & $\begin{array}{c}C_{5} \\
(\mathrm{cmH} 2 \mathrm{O})\end{array}$ & $\begin{array}{c}C_{6} \\
(k g)\end{array}$ & $\begin{array}{c}C_{7} \\
\text { (inch) }\end{array}$ & $\begin{array}{c}C_{8} \\
(\min )\end{array}$ \\
\hline$A_{1}$ & 0.1550 & 0.0383 & 0,0432 & 0.0598 & 0.041 & 0.0229 & 0.0217 & 0.0392 \\
\hline$A_{2}$ & 0.1654 & 0.0409 & 0.0432 & 0.0478 & 0.0455 & 0.0229 & 0.0217 & 0.0196 \\
\hline$A_{3}$ & 0.1395 & 0.046 & 0.0432 & 0.0478 & 0.0455 & 0.031 & 0.0217 & 0.0392 \\
\hline$A_{4}$ & 0.1499 & 0.046 & 0.041 & 0.0717 & 0.0455 & 0.017 & 0.0246 & 0.0196 \\
\hline$A_{5}$ & 0.1706 & 0.051 & 0.0519 & 0.0957 & 0.0455 & 0.014 & 0.0217 & 0.0784 \\
\hline$V^{+}$ & 0.1395 & 0.051 & 0.0519 & 0.0957 & 0.0455 & 0.014 & 0.0246 & 0.0784 \\
\hline $\boldsymbol{V}^{-}$ & 0.1706 & 0.0383 & 0.041 & 0.0478 & 0.041 & 0.031 & 0.0217 & 0.0196 \\
\hline
\end{tabular}

Calculated the Positive Ideal Distance $\left(\boldsymbol{M}_{\boldsymbol{i}}^{+}\right)$, Ideal Negative Distance $\left(\boldsymbol{M}_{\boldsymbol{i}}^{-}\right)$and performance score $\left(\boldsymbol{P}_{\boldsymbol{i}}\right)$ by using the above equation (6, 7 and 8) respectively. As seen in Fig. 1 the higher performance score indicate the best Mechanical Ventilator

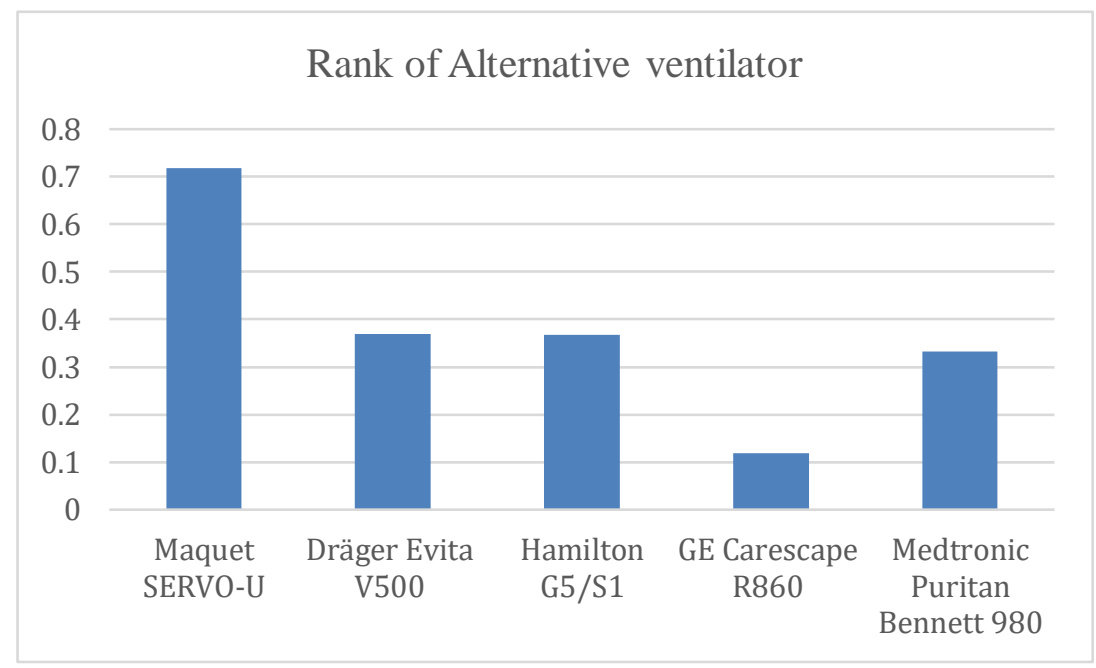

Fig. 1. Rank of alternative ventilator using TOPSIS technique

\subsection{PROMETHEE technique}

To verify the results for TOPSIS technique in the above, we applied PROMETHEE technique. PROMETHEE technique have two types PROMETHEE I and II. Determining the leaving and entering outranking flows of alternative will be done when using PROMETHEE I and this is called partial ranking of alternative. But when determining the net outranking flow for the alternative will be done by using PROMETHEE II, and this called the full ordering of alternative. PROMETHHE is consider one of the most widely used multi-criteria decision-making approaches, as it was used for the first time 
by Brans et al [15]. The PROMETHEE I technique introduced for the alternative partial ranking, while the PROMETHEE II technique for the alternative complete ordering.

PROMETHEE is another type of Multi criteria decision making. When using the PROMETHEE technique, decision makers assume the ratio of the weights of each criteria based on their information about the importance of each criteria, as this technique does not define nor give guidance for determining the weights of the criteria. In this review [16], some writers suggested that when using the PROMETHEE I, decision makers should participate in some additional effort, which is that some poor-performing alternatives can be excluded from the additional evaluation criteria, which may lead to reduced data requirements. While PROMETHEE II provides alternatives complete ranking, from best to worst, it is imperative to provide and inform decision-makers with information on how to change the final arrangement when making decisions of various criteria and weights. In this study we used the same weights criteria's in the above.

Below find a detailed step-by-step of the calculations, definitions and equations of the PROMETHEE II methodology taken from [17, 18], and the methodology was adopted in the numerical example to rank the alternatives and to choose the best mechanical ventilator device.

Step (1): The weight for each criteria must be determined,

$$
\sum_{j=1}^{n} W_{j}=1
$$

where $\mathrm{n}$ is the number of criteria

Step (2): Determine normalize the evaluation matrix, by using the equation $(10,11)$ respectively.

$$
N_{i j}=\frac{\left[x_{i j}-\min \left(x_{i j}\right)\right]}{\left[\max \left(x_{i j}\right)-\min \left(x_{i j}\right)\right]}
$$

for Beneficial criteria $(i=1,2,3, \ldots, m ; j=1,2,3, \ldots, n)$

$$
N_{i j}=\frac{\left[\max \left(x_{i j}\right)-x_{i j}\right]}{\left[\max \left(x_{i j}\right)-\min \left(x_{i j}\right)\right]}
$$

for Non-beneficial criteria $(i=1,2,3, \ldots, m ; j=1,2, ., n)$

Step (3): Determine the differences between any two alternatives.

Step (4): The preference function, $P_{j}(A, B)$ of each criteria $\mathrm{j}$ should be calculated by using equation (12).

$$
\begin{array}{r}
P_{j}(A, B)=0 \quad \text { if } N_{A j} \leq N_{B j} \\
P_{j}(A, B)=\left(N_{A j}-N_{B j}\right) \quad \text { if } N_{A j}>N_{B j}
\end{array}
$$

Where $\mathrm{a}$ and $\mathrm{b}$ denotes the alternatives

Step (5): The aggregated preference function, $\pi(A, B)$ should be calculated using the formula (13)

$$
\pi(A, B)=\left[\sum_{j=1}^{n} w_{j} P_{j}(A, B)\right] / \sum_{j=1}^{n} W_{j}
$$


Step (6): Next, the leaving and the entering outranking flows should be calculated using equations number $(14,15)$ respectively;

$$
\begin{array}{ll}
\frac{1}{m-1} \sum_{b=1}^{m} \pi(A, B) & (A \neq B) \\
\frac{1}{m-1} \sum_{b=1}^{m} \pi(B, A) & (A \neq B)
\end{array}
$$

Where $m$ denotes the alternative

Step (7): The net outranking flow for each alternative $\Phi^{\text {net }}$ should be calculated using equation (16)

$$
\Phi^{n e t}=\Phi^{+}-\Phi^{-}
$$

Finally, the alternative ranking according the net outranking flow $\Phi^{\text {net }}$ for each value. The best alternative is the higher of net outranking flow $\Phi^{\text {net }}$.

The same previous data was used to choosing and evaluating the alternative by applying the PROMETHEE II technique and comparing the results obtained through the PROMETHEE II technique with the results obtained through the TOPSIS technique.

All steps of the PROMETHEE II technique are applied as shown in the following

\begin{tabular}{|c|c|c|c|c|c|c|c|c|}
\hline & $\begin{array}{c}\text { Non } \\
\text { Beneficial } \\
\text { Criteria }\end{array}$ & $\begin{array}{c}\text { Beneficial } \\
\text { Criteria }\end{array}$ & $\begin{array}{c}\text { Beneficial } \\
\text { Criteria }\end{array}$ & $\begin{array}{c}\text { Beneficial } \\
\text { Criteria }\end{array}$ & $\begin{array}{c}\text { Beneficial } \\
\text { Criteria }\end{array}$ & $\begin{array}{l}\text { Non Bene- } \\
\text { ficial Cri- } \\
\text { teria }\end{array}$ & \begin{tabular}{|c|} 
Beneficial \\
Criteria
\end{tabular} & $\begin{array}{c}\text { Beneficial } \\
\text { Criteria }\end{array}$ \\
\hline & $\begin{array}{l}C_{1} \\
(\$)\end{array}$ & $\begin{array}{c}C_{2} \\
(l / m i n)\end{array}$ & $\begin{array}{c}C_{3} \\
(\mathrm{~cm} H 2 O)\end{array}$ & $\begin{array}{c}C_{4} \\
(m l)\end{array}$ & $\begin{array}{c}C_{5} \\
(\mathrm{cmH} 2 \mathrm{O})\end{array}$ & $\begin{array}{c}C_{6} \\
(\mathrm{~kg})\end{array}$ & $\begin{array}{c}C_{7} \\
\text { (inch) }\end{array}$ & $\begin{array}{c}C_{8} \\
(\min )\end{array}$ \\
\hline$A_{1}$ & 30,000 & 150 & 100 & $2-2500$ & $0-45$ & 31 & 15 & 60 \\
\hline$A_{2}$ & 32,000 & 160 & 100 & $2-2000$ & $1-50$ & 31 & 15 & 30 \\
\hline$A_{3}$ & 27,000 & 180 & 100 & $2-2000$ & $0-50$ & 42 & 15 & 60 \\
\hline$A_{4}$ & 29,000 & 180 & 95 & $2-3000$ & $0-50$ & 23 & 17 & 30 \\
\hline$A_{5}$ & 33,000 & 200 & 120 & $2-4000$ & $1-50$ & 19 & 15 & 120 \\
\hline $\begin{array}{l}\text { Max } \\
\left(\boldsymbol{X}_{i j}\right)\end{array}$ & 33,000 & 200 & 120 & 4000 & 50 & 42 & 17 & 120 \\
\hline $\begin{array}{l}\text { Min } \\
\left(\boldsymbol{X}_{\boldsymbol{i} \boldsymbol{j}}\right)\end{array}$ & 27,000 & 150 & 95 & 2000 & 45 & 19 & 15 & 30 \\
\hline
\end{tabular}
Tables.

Table 5. Maximum and minimum value for each criteria 
Table 6. Calculated $\boldsymbol{R}_{\boldsymbol{i j}}$ for beneficial and non beneficial criteria

\begin{tabular}{|l|c|c|c|c|c|c|c|c|}
\hline & $\begin{array}{c}\text { Non } \\
\text { Beneficial } \\
\text { Criteria }\end{array}$ & $\begin{array}{c}\text { Beneficial } \\
\text { Criteria }\end{array}$ & $\begin{array}{c}\text { Beneficial } \\
\text { Criteria }\end{array}$ & $\begin{array}{c}\text { Beneficial } \\
\text { Criteria }\end{array}$ & $\begin{array}{c}\text { Beneficial } \\
\text { Criteria }\end{array}$ & $\begin{array}{c}\text { Non } \\
\text { Beneficial } \\
\text { Criteria }\end{array}$ & $\begin{array}{c}\text { Beneficial } \\
\text { Criteria }\end{array}$ & $\begin{array}{c}\text { Beneficial } \\
\text { Criteria }\end{array}$ \\
\hline & $\begin{array}{c}\boldsymbol{C}_{\mathbf{1}} \\
(\$)\end{array}$ & $\begin{array}{c}\boldsymbol{C}_{\mathbf{2}} \\
(\mathbf{l} \text { min })\end{array}$ & $\begin{array}{c}\boldsymbol{C}_{\mathbf{3}} \\
(\mathbf{c m} \boldsymbol{H} 2 \boldsymbol{O})\end{array}$ & $\begin{array}{c}\boldsymbol{C}_{\mathbf{4}} \\
(\boldsymbol{m l})\end{array}$ & $\begin{array}{c}\boldsymbol{C}_{\mathbf{5}} \\
(\mathbf{c m} \boldsymbol{H} 2 \boldsymbol{O})\end{array}$ & $\begin{array}{c}\boldsymbol{C}_{\mathbf{6}} \\
(\mathbf{k g})\end{array}$ & $\begin{array}{c}\boldsymbol{C}_{\mathbf{7}} \\
(\text { inch })\end{array}$ & $\begin{array}{c}\boldsymbol{C}_{\mathbf{8}} \\
(\boldsymbol{m i n})\end{array}$ \\
\hline $\boldsymbol{A}_{\mathbf{1}}$ & 0.5 & 0 & 0.2 & 0.25 & 0.0 & 0.478 & 0.0 & 0.333 \\
\hline $\boldsymbol{A}_{\mathbf{2}}$ & 0.166 & 0.2 & 0.2 & 0.0 & 1 & 0.478 & 0.0 & 0.0 \\
\hline $\boldsymbol{A}_{\mathbf{3}}$ & 1 & 0.6 & 0.2 & 0.0 & 1 & 0.0 & 0.0 & 0.333 \\
\hline $\boldsymbol{A}_{\mathbf{4}}$ & 0.666 & 0.6 & 0.0 & 0.5 & 1 & 0.826 & 1 & 0.0 \\
\hline $\boldsymbol{A}_{\mathbf{5}}$ & 0.0 & 1 & 1 & 1 & 1 & 1 & 0.0 & 1 \\
\hline
\end{tabular}

Table 7. Calculated the evaluate differences of each two alternative

\begin{tabular}{|c|c|c|c|c|c|c|c|c|}
\hline & $\begin{array}{c}\boldsymbol{C}_{\mathbf{1}} \\
\mathbf{( \$ )}\end{array}$ & $\begin{array}{c}\boldsymbol{C}_{\mathbf{2}} \\
(\mathbf{1 / m i n})\end{array}$ & $\begin{array}{c}\boldsymbol{C}_{\mathbf{3}} \\
(\mathbf{c m} \mathbf{H} 2 \mathbf{O})\end{array}$ & $\begin{array}{c}\boldsymbol{C}_{\mathbf{4}} \\
(\mathbf{m l})\end{array}$ & $\begin{array}{c}\boldsymbol{C}_{\mathbf{5}} \\
(\mathbf{c m} \mathbf{H} \mathbf{O})\end{array}$ & $\begin{array}{c}\boldsymbol{C}_{\mathbf{6}} \\
(\mathbf{k g})\end{array}$ & $\begin{array}{c}\boldsymbol{C}_{\mathbf{7}} \\
(\mathbf{i n c h})\end{array}$ & $\begin{array}{c}\boldsymbol{C}_{\mathbf{8}} \\
(\mathbf{m i n})\end{array}$ \\
\hline $\boldsymbol{A}_{\mathbf{1}}$ & 0.5 & 0 & 0.2 & 0.25 & 0.0 & 0.478 & 0.0 & 0.333 \\
\hline $\boldsymbol{A}_{\mathbf{2}}$ & 0.166 & 0.2 & 0.2 & 0.0 & 1 & 0.478 & 0.0 & 0.0 \\
\hline $\boldsymbol{A}_{\mathbf{3}}$ & 1 & 0.6 & 0.2 & 0.0 & 1 & 0.0 & 0.0 & 0.333 \\
\hline $\boldsymbol{A}_{\mathbf{4}}$ & 0.666 & 0.6 & 0.0 & 0.5 & 1 & 0.826 & 1 & 0.0 \\
\hline $\boldsymbol{A}_{\mathbf{5}}$ & 0.0 & 1 & 1 & 1 & 1 & 1 & 0.0 & 1 \\
\hline $\mathrm{D}\left(\boldsymbol{A}_{\mathbf{1}}-\boldsymbol{A}_{\mathbf{2}}\right)$ & 0.334 & -0.2 & 0 & 0.25 & -1 & 0 & 0 & 0.333 \\
\hline $\mathrm{D}\left(\boldsymbol{A}_{\mathbf{1}}-\boldsymbol{A}_{\mathbf{3}}\right)$ & -0.5 & -0.6 & 0 & 0.25 & -1 & 0.478 & 0 & 0 \\
\hline $\mathrm{D}\left(\boldsymbol{A}_{\mathbf{1}}-\boldsymbol{A}_{\mathbf{4}}\right)$ & -0.166 & -0.6 & 0.2 & -0.25 & -1 & -0.348 & -1 & 0.333 \\
\hline $\mathrm{D}\left(\boldsymbol{A}_{\mathbf{1}}-\boldsymbol{A}_{\mathbf{5}}\right)$ & 0.5 & -1 & -0.8 & 0.25 & -1 & -0.522 & 0 & -0.667 \\
\hline $\mathrm{D}\left(\boldsymbol{A}_{\mathbf{2}}-\boldsymbol{A}_{\mathbf{1}}\right)$ & -0.334 & 0.2 & 0 & -0.75 & 1 & 0 & 0 & -0.333 \\
\hline $\mathrm{D}\left(\boldsymbol{A}_{\mathbf{2}}-\boldsymbol{A}_{\mathbf{3}}\right)$ & -0.834 & -0.4 & 0 & 0 & 0 & 0.478 & 0 & -0.333 \\
\hline $\mathrm{D}\left(\boldsymbol{A}_{\mathbf{2}}-\boldsymbol{A}_{\mathbf{4}}\right)$ & -0.5 & -0.4 & 0.2 & -0.5 & 0 & -0.348 & -1 & 0 \\
\hline $\mathrm{D}\left(\boldsymbol{A}_{\mathbf{2}}-\boldsymbol{A}_{\mathbf{5}}\right)$ & 0.166 & -0.8 & -0.8 & -1 & 0 & -0.522 & 0 & -1 \\
\hline $\mathrm{D}\left(\boldsymbol{A}_{\mathbf{3}}-\boldsymbol{A}_{\mathbf{1}}\right)$ & 0.5 & 0.6 & 0 & -0.25 & 1 & -0.478 & 0 & 0 \\
\hline $\mathrm{D}\left(\boldsymbol{A}_{\mathbf{3}}-\boldsymbol{A}_{\mathbf{2}}\right)$ & 0.834 & 0.4 & 0 & 0 & 0 & -0.478 & 0 & 0.333 \\
\hline $\mathrm{D}\left(\boldsymbol{A}_{\mathbf{3}}-\boldsymbol{A}_{\mathbf{4}}\right)$ & 0.334 & 0 & 0.2 & -0.5 & 0 & -0.826 & -1 & 0333 \\
\hline $\mathrm{D}\left(\boldsymbol{A}_{\mathbf{3}}-\boldsymbol{A}_{\mathbf{5}}\right)$ & 1 & -0.4 & -0.8 & -1 & 0 & -1 & 0 & -0.667 \\
\hline $\mathrm{D}\left(\boldsymbol{A}_{\mathbf{4}}-\boldsymbol{A}_{\mathbf{1}}\right)$ & 0.166 & 0.6 & -0.2 & 0.25 & 1 & 0.348 & 1 & -0.333 \\
\hline $\mathrm{D}\left(\boldsymbol{A}_{\mathbf{4}}-\boldsymbol{A}_{\mathbf{2}}\right)$ & 0.5 & 0.4 & -0.2 & 0.5 & 0 & 0.348 & 1 & 0 \\
\hline $\mathrm{D}\left(\boldsymbol{A}_{\mathbf{4}}-\boldsymbol{A}_{\mathbf{3}}\right)$ & -0.334 & 0 & -0.2 & 0.5 & 0 & 0.826 & 1 & -0.333 \\
\hline $\mathrm{D}\left(\boldsymbol{A}_{\mathbf{4}}-\boldsymbol{A}_{\mathbf{5}}\right)$ & 0.666 & -0.4 & -1 & -0.5 & 0 & -0.174 & 1 & -1 \\
\hline $\mathrm{D}\left(\boldsymbol{A}_{\mathbf{5}}-\boldsymbol{A}_{\mathbf{1}}\right)$ & -0.5 & 1 & 0.8 & 0.75 & 1 & 0.522 & 0 & 0.667 \\
\hline $\mathrm{D}\left(\boldsymbol{A}_{\mathbf{5}}-\boldsymbol{A}_{\mathbf{2}}\right)$ & -0.166 & 0.8 & 0.8 & 1 & 0 & 0.522 & 0 & 1 \\
\hline $\mathrm{D}\left(\boldsymbol{A}_{\mathbf{5}}-\boldsymbol{A}_{\mathbf{3}}\right)$ & -1 & 0.4 & 0.8 & 1 & 0 & 1 & 0 & 0.667 \\
\hline $\mathrm{D}\left(\boldsymbol{A}_{\mathbf{5}}-\boldsymbol{A}_{\mathbf{4}}\right)$ & -0.666 & 0.4 & 1 & 0.5 & 0 & 0.174 & -1 & 1 \\
\hline & & & & & & & & \\
\hline
\end{tabular}


Table 8. Calculate the preference function $P_{j}(a, b)$ of each criteria

\begin{tabular}{|c|c|c|c|c|c|c|c|c|}
\hline & $\begin{array}{c}\boldsymbol{C}_{\mathbf{1}} \\
(\mathbf{\$})\end{array}$ & $\begin{array}{c}\boldsymbol{C}_{\mathbf{2}} \\
(\mathbf{I} / \mathbf{m i n})\end{array}$ & $\begin{array}{c}\boldsymbol{C}_{\mathbf{3}} \\
(\mathbf{c m H} 2 \mathbf{O})\end{array}$ & $\begin{array}{c}\boldsymbol{C}_{\mathbf{4}} \\
(\mathbf{m l})\end{array}$ & $\begin{array}{c}\boldsymbol{C}_{\mathbf{5}} \\
(\mathbf{c m H 2})\end{array}$ & $\begin{array}{c}\boldsymbol{C}_{\mathbf{6}} \\
(\mathbf{k g})\end{array}$ & $\begin{array}{c}\boldsymbol{C}_{\mathbf{7}} \\
(\mathbf{i n c h})\end{array}$ & $\begin{array}{c}\boldsymbol{C}_{\mathbf{8}} \\
(\mathbf{m i n})\end{array}$ \\
\hline $\mathrm{P}\left(\boldsymbol{A}_{\mathbf{1}}-\boldsymbol{A}_{\mathbf{2}}\right)$ & 0.334 & 0 & 0 & 0.25 & 0 & 0 & 0 & 0.333 \\
\hline $\mathrm{P}\left(\boldsymbol{A}_{\mathbf{1}}-\boldsymbol{A}_{\mathbf{3}}\right)$ & 0 & 0 & 0 & 0.25 & 0 & 0.478 & 0 & 0 \\
\hline $\mathrm{P}\left(\boldsymbol{A}_{\mathbf{1}}-\boldsymbol{A}_{\mathbf{4}}\right)$ & 0 & 0 & 0.2 & 0 & 0 & 0 & 0 & 0.333 \\
\hline $\mathrm{P}\left(\boldsymbol{A}_{\mathbf{1}}-\boldsymbol{A}_{\mathbf{5}}\right)$ & 0.5 & 0 & 0 & 0.25 & 0 & 0 & 0 & 0 \\
\hline $\mathrm{P}\left(\boldsymbol{A}_{\mathbf{2}}-\boldsymbol{A}_{\mathbf{1}}\right)$ & 0 & 0.2 & 0 & 0 & 1 & 0 & 0 & 0 \\
\hline $\mathrm{P}\left(\boldsymbol{A}_{\mathbf{2}}-\boldsymbol{A}_{\mathbf{3}}\right)$ & 0 & 0 & 0 & 0 & 0 & 0.478 & 0 & 0 \\
\hline $\mathrm{P}\left(\boldsymbol{A}_{\mathbf{2}}-\boldsymbol{A}_{\mathbf{4}}\right)$ & 0 & 0 & 0.2 & 0 & 0 & 0 & 0 & 0 \\
\hline $\mathrm{P}\left(\boldsymbol{A}_{\mathbf{2}}-\boldsymbol{A}_{\mathbf{5}}\right)$ & 0.166 & 0 & 0 & 0 & 0 & 0 & 0 & 0 \\
\hline $\mathrm{P}\left(\boldsymbol{A}_{\mathbf{3}}-\boldsymbol{A}_{\mathbf{1}}\right)$ & 0.5 & 0.6 & 0 & 0 & 1 & 0 & 0 & 0 \\
\hline $\mathrm{P}\left(\boldsymbol{A}_{\mathbf{3}}-\boldsymbol{A}_{\mathbf{2}}\right)$ & 0.834 & 0.4 & 0 & 0 & 0 & 0 & 0 & 0.333 \\
\hline $\mathrm{P}\left(\boldsymbol{A}_{\mathbf{3}}-\boldsymbol{A}_{\mathbf{4}}\right)$ & 0.334 & 0 & 0.2 & 0 & 0 & 0 & 0 & 0333 \\
\hline $\mathrm{P}\left(\boldsymbol{A}_{\mathbf{3}}-\boldsymbol{A}_{\mathbf{5}}\right)$ & 1 & 0 & 0 & 0 & 0 & 0 & 0 & 0 \\
\hline $\mathrm{P}\left(\boldsymbol{A}_{\mathbf{4}}-\boldsymbol{A}_{\mathbf{1}}\right)$ & 0.166 & 0.6 & 0 & 0.25 & 1 & 0.348 & 1 & 0 \\
\hline $\mathrm{P}\left(\boldsymbol{A}_{\mathbf{4}}-\boldsymbol{A}_{\mathbf{2}}\right)$ & 0.5 & 0.4 & 0 & 0.5 & 0 & 0.348 & 1 & 0 \\
\hline $\mathrm{P}\left(\boldsymbol{A}_{\mathbf{4}}-\boldsymbol{A}_{\mathbf{3}}\right)$ & 0 & 0 & 0 & 0.5 & 0 & 0.826 & 1 & 0 \\
\hline $\mathrm{P}\left(\boldsymbol{A}_{\mathbf{4}}-\boldsymbol{A}_{\mathbf{5}}\right)$ & 0.666 & 0 & 0 & 0 & 0 & 0 & 1 & 0 \\
\hline $\mathrm{P}\left(\boldsymbol{A}_{\mathbf{5}}-\boldsymbol{A}_{\mathbf{1}}\right)$ & 0 & 1 & 0.8 & 0.75 & 1 & 0.522 & 0 & 0.667 \\
\hline $\mathrm{P}\left(\boldsymbol{A}_{\mathbf{5}}-\boldsymbol{A}_{\mathbf{2}}\right)$ & 0 & 0.8 & 0.8 & 1 & 0 & 0.522 & 0 & 1 \\
\hline $\mathrm{P}\left(\boldsymbol{A}_{\mathbf{5}}-\boldsymbol{A}_{\mathbf{3}}\right)$ & 0 & 0.4 & 0.8 & 1 & 0 & 1 & 0 & 0.667 \\
\hline $\mathrm{P}\left(\boldsymbol{A}_{\mathbf{5}}-\boldsymbol{A}_{\mathbf{4}}\right)$ & 0 & 0.4 & 1 & 0.5 & 0 & 0.174 & 0 & 1 \\
\hline
\end{tabular}

Table 9. Calculated the aggregated preference function

\begin{tabular}{|c|c|c|c|c|c|c|c|c|}
\hline & 0.35 & 0.1 & 0.1 & 0.15 & 0.1 & 0.05 & 0.05 & 0.1 \\
\hline & $\begin{array}{l}C_{1} \\
(\$)\end{array}$ & $\begin{array}{c}\boldsymbol{C}_{2} \\
(1 / \mathrm{min}) \\
\end{array}$ & $\begin{array}{c}\boldsymbol{C}_{3} \\
(\mathrm{cmH} 2 \mathrm{O})\end{array}$ & $\begin{array}{c}\boldsymbol{C}_{\boldsymbol{4}} \\
(\mathrm{ml})\end{array}$ & $\begin{array}{c}\boldsymbol{C}_{\mathbf{5}} \\
(\mathrm{cmH} 2 \mathrm{O})\end{array}$ & $\begin{array}{c}\boldsymbol{C}_{6} \\
(\mathrm{~kg})\end{array}$ & $\begin{array}{c}C_{7} \\
\text { (inch) }\end{array}$ & $\begin{array}{c}\boldsymbol{C}_{\mathbf{8}} \\
(\mathrm{min})\end{array}$ \\
\hline$w_{j} * \mathrm{P}\left(A_{1}-A_{2}\right)$ & 0.1169 & 0 & 0 & 0.0375 & 0 & 0 & 0 & 0.0333 \\
\hline$w_{j} * \mathrm{P}\left(A_{1}-A_{3}\right)$ & 0 & 0 & 0 & 0.0375 & 0 & 0.0239 & 0 & 0 \\
\hline $\boldsymbol{w}_{j} * \mathrm{P}\left(\boldsymbol{A}_{1}-\boldsymbol{A}_{\mathbf{4}}\right)$ & 0 & 0 & 0.02 & 0 & 0 & 0 & 0 & 0.0333 \\
\hline$w_{j} * \mathrm{P}\left(A_{1}-A_{5}\right)$ & 0.175 & 0 & 0 & 0.0375 & 0 & 0 & 0 & 0 \\
\hline $\boldsymbol{w}_{j} * \mathrm{P}\left(\boldsymbol{A}_{\mathbf{2}}-\boldsymbol{A}_{\mathbf{1}}\right)$ & 0 & 0.02 & 0 & 0 & 0.1 & 0 & 0 & 0 \\
\hline$w_{j} * \mathrm{P}\left(A_{2}-A_{3}\right)$ & 0 & 0 & 0 & 0 & 0 & 0.0239 & 0 & 0 \\
\hline$w_{j} * \mathrm{P}\left(A_{2}-A_{4}\right)$ & 0 & 0 & 0.02 & 0 & 0 & 0 & 0 & 0 \\
\hline $\boldsymbol{w}_{\boldsymbol{j}} * \mathrm{P}\left(\boldsymbol{A}_{\mathbf{2}}-\boldsymbol{A}_{5}\right)$ & 0.0581 & 0 & 0 & 0 & 0 & 0 & 0 & 0 \\
\hline$w_{j} * \mathrm{P}\left(A_{3}-A_{1}\right)$ & 0.175 & 0.06 & 0 & 0 & 1 & 0 & 0 & 0 \\
\hline $\boldsymbol{w}_{j} * \mathrm{P}\left(\boldsymbol{A}_{3}-\boldsymbol{A}_{2}\right)$ & 0.2919 & 0.04 & 0 & 0 & 0 & 0 & 0 & 0.0333 \\
\hline$w_{j} * \mathrm{P}\left(A_{3}-A_{4}\right)$ & 0.1169 & 0 & 0.02 & 0 & 0 & 0 & 0 & 0.0333 \\
\hline$w_{j} * \mathrm{P}\left(A_{3}-A_{5}\right)$ & 0.35 & 0 & 0 & 0 & 0 & 0 & 0 & 0 \\
\hline$w_{j} * \mathrm{P}\left(A_{4}-A_{1}\right)$ & 0.0581 & 0.06 & 0 & 0.0375 & 0.1 & 0.0174 & 0.05 & 0 \\
\hline $\boldsymbol{w}_{\boldsymbol{j}} * \mathrm{P}\left(\boldsymbol{A}_{\mathbf{4}}-\boldsymbol{A}_{\mathbf{2}}\right)$ & 0.175 & 0.04 & 0 & 0.075 & 0 & 0.0174 & 0.05 & 0 \\
\hline $\boldsymbol{w}_{j} * \mathrm{P}\left(\boldsymbol{A}_{\mathbf{4}}-\boldsymbol{A}_{\mathbf{3}}\right)$ & 0 & 0 & 0 & 0.075 & 0 & 0.0413 & 0.05 & 0 \\
\hline $\boldsymbol{w}_{j} * \mathrm{P}\left(\boldsymbol{A}_{\mathbf{4}}-\boldsymbol{A}_{\mathbf{5}}\right)$ & 0.2331 & 0 & 0 & 0 & 0 & 0 & 0.05 & 0 \\
\hline$w_{j} * \mathrm{P}\left(A_{5}-A_{1}\right)$ & 0 & 0.1 & 0.08 & 0.1125 & 0.1 & 0.0261 & 0 & 0.0667 \\
\hline
\end{tabular}




\begin{tabular}{|c|c|c|c|c|c|c|c|c|}
\hline $\boldsymbol{w}_{\boldsymbol{j}} * \mathrm{P}\left(\boldsymbol{A}_{\mathbf{5}}-\boldsymbol{A}_{\mathbf{2}}\right)$ & 0 & 0.08 & 0.08 & 0.15 & 0 & 0.0261 & 0 & 0.1 \\
\hline $\boldsymbol{w}_{\boldsymbol{j}} * \mathrm{P}\left(\boldsymbol{A}_{\mathbf{5}}-\boldsymbol{A}_{\mathbf{3}}\right)$ & 0 & 0.04 & 0.08 & 0.15 & 0 & 0.05 & 0 & 0.0667 \\
\hline $\boldsymbol{w}_{\boldsymbol{j}} * \mathrm{P}\left(\boldsymbol{A}_{\mathbf{5}}-\boldsymbol{A}_{\mathbf{4}}\right)$ & 0 & 0.04 & 0.1 & 0.075 & 0 & 0.0087 & 0 & 0.1 \\
\hline & & & & & & & & \\
\hline
\end{tabular}

Table 10.

Calculated $\pi(a, b)$

\begin{tabular}{|c|c|}
\hline & $\pi(a, b)$ \\
\hline $\boldsymbol{w}_{j} * \mathrm{P}\left(\boldsymbol{A}_{1}-\boldsymbol{A}_{\mathbf{2}}\right)$ & 0.1877 \\
\hline $\boldsymbol{w}_{j} * \mathrm{P}\left(\boldsymbol{A}_{1}-\boldsymbol{A}_{3}\right)$ & 0.0614 \\
\hline $\boldsymbol{w}_{j} * \mathrm{P}\left(\boldsymbol{A}_{1}-\boldsymbol{A}_{4}\right)$ & 0.0533 \\
\hline $\boldsymbol{w}_{\boldsymbol{j}} * \mathrm{P}\left(\boldsymbol{A}_{\mathbf{1}}-\boldsymbol{A}_{\mathbf{5}}\right)$ & 0.2125 \\
\hline$w_{j} * \mathrm{P}\left(A_{2}-A_{1}\right)$ & 0.12 \\
\hline $\boldsymbol{w}_{\boldsymbol{j}} * \mathrm{P}\left(\boldsymbol{A}_{2}-\boldsymbol{A}_{3}\right)$ & 0.0239 \\
\hline $\boldsymbol{w}_{j} * \mathrm{P}\left(\boldsymbol{A}_{2}-\boldsymbol{A}_{\mathbf{4}}\right)$ & 0.02 \\
\hline $\boldsymbol{w}_{\boldsymbol{j}} * \mathrm{P}\left(\boldsymbol{A}_{\mathbf{2}}-\boldsymbol{A}_{\mathbf{5}}\right)$ & 0.0581 \\
\hline $\boldsymbol{w}_{j} * \mathrm{P}\left(\boldsymbol{A}_{3}-\boldsymbol{A}_{1}\right)$ & 0.235 \\
\hline $\boldsymbol{w}_{\boldsymbol{j}} * \mathrm{P}\left(\boldsymbol{A}_{\mathbf{3}}-\boldsymbol{A}_{\mathbf{2}}\right)$ & 0.3652 \\
\hline $\boldsymbol{w}_{j} * \mathrm{P}\left(\boldsymbol{A}_{3}-\boldsymbol{A}_{4}\right)$ & 0.1702 \\
\hline $\boldsymbol{w}_{\boldsymbol{j}} * \mathrm{P}\left(\boldsymbol{A}_{\mathbf{3}}-\boldsymbol{A}_{\mathbf{5}}\right)$ & 0.35 \\
\hline $\boldsymbol{w}_{\boldsymbol{j}} * \mathrm{P}\left(\boldsymbol{A}_{\mathbf{4}}-\boldsymbol{A}_{\mathbf{1}}\right)$ & 0.323 \\
\hline $\boldsymbol{w}_{\boldsymbol{j}} * \mathrm{P}\left(\boldsymbol{A}_{\mathbf{4}}-\boldsymbol{A}_{\mathbf{2}}\right)$ & 0.3574 \\
\hline $\boldsymbol{w}_{j} * \mathrm{P}\left(\boldsymbol{A}_{\mathbf{4}}-\boldsymbol{A}_{3}\right)$ & 0.1663 \\
\hline $\boldsymbol{w}_{\boldsymbol{j}} * \mathrm{P}\left(\boldsymbol{A}_{\mathbf{4}}-\boldsymbol{A}_{\mathbf{5}}\right)$ & 0.2831 \\
\hline $\boldsymbol{w}_{\boldsymbol{j}} * \mathrm{P}\left(\boldsymbol{A}_{\mathbf{5}}-\boldsymbol{A}_{\mathbf{1}}\right)$ & 0.4853 \\
\hline $\boldsymbol{w}_{j} * \mathrm{P}\left(\boldsymbol{A}_{\mathbf{5}}-\boldsymbol{A}_{\mathbf{2}}\right)$ & 0.4361 \\
\hline $\boldsymbol{w}_{j} * \mathrm{P}\left(\boldsymbol{A}_{\mathbf{5}}-\boldsymbol{A}_{\mathbf{3}}\right)$ & 0.3867 \\
\hline $\boldsymbol{w}_{j} * \mathrm{P}\left(\boldsymbol{A}_{\mathbf{5}}-\boldsymbol{A}_{\mathbf{4}}\right)$ & 0.3237 \\
\hline
\end{tabular}

Table 11. Determine the aggregated preference matrix

\begin{tabular}{|c|c|c|c|c|c|}
\hline $\begin{array}{c}\text { Aggregated prefer- } \\
\text { ence function }\end{array}$ & $\boldsymbol{A}_{\mathbf{1}}$ & $\boldsymbol{A}_{\mathbf{2}}$ & $\boldsymbol{A}_{\mathbf{3}}$ & $\boldsymbol{A}_{\mathbf{4}}$ & $\boldsymbol{A}_{\mathbf{5}}$ \\
\hline $\boldsymbol{A}_{\mathbf{1}}$ & - & 0.1877 & 0.0614 & 0.0533 & 0.2125 \\
\hline $\boldsymbol{A}_{\mathbf{2}}$ & 0.12 & - & 0.0239 & 0.02 & 0.0581 \\
\hline $\boldsymbol{A}_{\mathbf{3}}$ & 0.235 & 0.3652 & - & 0.1822 & 0.35 \\
\hline $\boldsymbol{A}_{\mathbf{4}}$ & 0.323 & 0.3574 & 0.1663 & - & 0.2831 \\
\hline $\boldsymbol{A}_{\mathbf{5}}$ & 0.4853 & 0.4361 & 0.3867 & 0.3237 & - \\
\hline
\end{tabular}


Table 12. Determine the leaving and the entering outranking flows

\begin{tabular}{|c|c|c|c|c|c|c|}
\hline $\begin{array}{c}\text { Aggregated prefer- } \\
\text { ence function }\end{array}$ & $\boldsymbol{A}_{\mathbf{1}}$ & $\boldsymbol{A}_{\mathbf{2}}$ & $\boldsymbol{A}_{\mathbf{3}}$ & $\boldsymbol{A}_{\mathbf{4}}$ & $\boldsymbol{A}_{\mathbf{5}}$ & $\begin{array}{c}\boldsymbol{\Phi}^{+} \\
\text {Leaving } \text { flow }\end{array}$ \\
\hline $\boldsymbol{A}_{\mathbf{1}}$ & - & 0.1877 & 0.0614 & 0.0533 & 0.2125 & 0.1287 \\
\hline $\boldsymbol{A}_{\mathbf{2}}$ & 0.12 & - & 0.0239 & 0.02 & 0.0581 & 0.0555 \\
\hline $\boldsymbol{A}_{\mathbf{3}}$ & 0.235 & 0.3652 & - & 0.1822 & 0.35 & 0.2831 \\
\hline $\boldsymbol{A}_{\mathbf{4}}$ & 0.323 & 0.3574 & 0.1663 & - & 0.2831 & 0.2824 \\
\hline$A_{5}$ & 0.4853 & 0.4361 & 0.3867 & 0.3237 & - & 0.4079 \\
\hline $\begin{array}{c}\boldsymbol{\Phi}^{-} \\
\text {Entering flow }\end{array}$ & 0.2908 & 0.3366 & 0.1595 & 0.1448 & 0.2259 & \\
\hline
\end{tabular}

Calculated the net outranking flow for each alternative $\Phi^{\text {net }}$ by using the above equation (16). Rank the alternative based of the outranking flow. As see in Fig. 2 the higher outranking flow is the best alternative

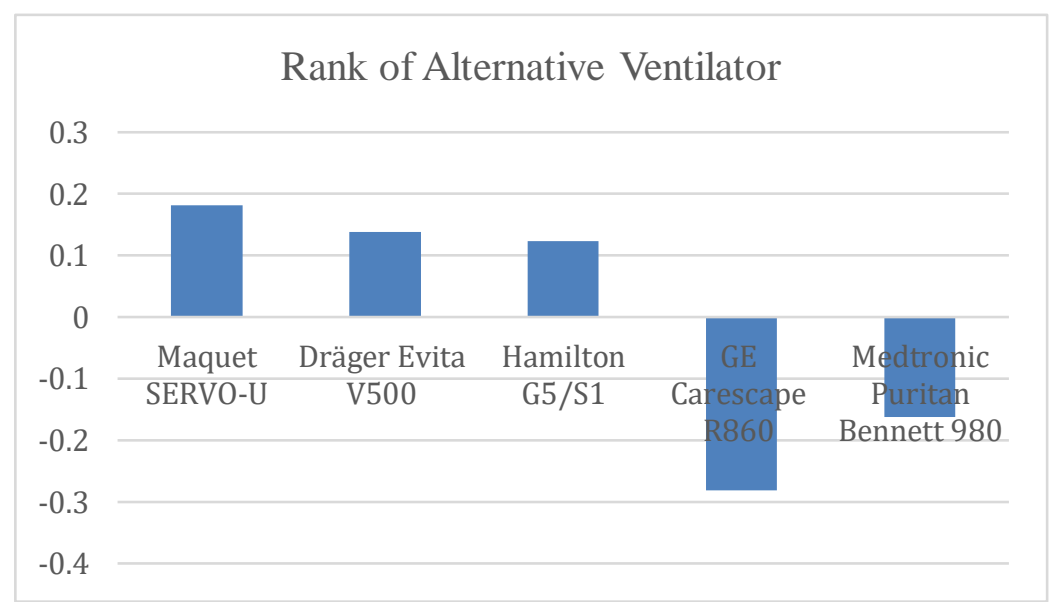

Fig. 2. Rank of alternative ventilator using PROMETHEE technique

\section{$4 \quad$ Results}

After applied Multi Criteria Decision Making (MCDM) methods to evaluating some of alternatives mechanical ventilator equipment's and to selecting the best mechanical ventilator device from among alternatives by using TOPSIS technique and PROMETHEE II technique. As the results in Fig. 1 and Fig. 2 MAQUET servo U ventilator is the first rank \& it is the highest price device; Dräger Evita V500 in the second rank; then in the third rank is Hamilton G5/S1; Medtronic Puritan Bennett 980 in the fourth rank and in the last rank is GE Carescape R860. The patient's stay on the ventilator for a long time leads to damage to a healthy lung. This method helped medical engineers and decision-makers to choose ventilator with standards aimed at patient comfort and aid in his recovery within a shorter period than traditional ventilator.

MAQUET servo U ventilator is the best choice mechanical ventilator among the five alternatives based on eight criteria was selected according to the study. MAQUET servo 
U can reduces the time of patient stays on the ventilator. MAQUET Servo U ventilator is the best and optimal device as its having the maximum performance value. This result may be a recommendation to purchase a mechanical ventilator.

\section{Discussion}

Multi-criteria decision-making (MCDM) techniques can be applied to select the best ventilator. In this study, we propose TOPSIS technique \& PROMETHEE technique for selecting and evaluating ventilators.

The proposed new method will provide a practical solution in medical equipment supply. In this study, weights were determined for each criteria and the result was reached after evaluating the alternatives for ventilators.

This methodology indicated that the MAQUET servo $U$ ventilator is the best mechanical ventilator when applied to the TOPSIS technique \& PROMETHEE technique although the MAQUET servo $U$ ventilator is the most expensive in price. The criteria and weights of each criteria is given care by the medical experts and decision-makers. MCDM techniques help intensive care physicians, medical engineers, and users to choose the best ventilator. According to the opinion of medical engineers and hospital decision-makers, important criteria such as cost of the MV device; maxi-mum inspiratory flow; maximum pressure; tidal volume; PEEP; the weight of ventilator; screen size, and internal battery time. These results are consistent with the findings of Rogalewicz and Jurickova [19] which was used the MCDM technique in their study. Ventilators must be provided to the patients in the intensive care units (ICU) with high quality. A ventilator helps the patient to breathe, so it can help the patient recover as quickly as possible, and not be a cause of damage to a healthy lung [20]. Choosing a low-efficiency ventilator can lead to damage to a healthy lung in addition to other damage due to the long period of patient contact with the ventilator [21].

The current study will help to assist medical engineers, doctors, and decision-makers in making the appropriate decision to select the best ventilator. In addition, the proposed new method also can provide solutions to decision-making problems in the medical field of multiple criteria and in the purchasing of medical devices.

\section{Conclusion}

Based on the above results, a methodology has been designed to evaluate of the mechanical ventilator prior to their purchase. This methodology can serve as support for relevant decisions. This methodology and the appropriateness of using this technique were discussed with some experts in hospitals, and it was accepted very positively, and it should be note that the process of purchasing a respirator is linked to collecting information on some important criteria's through suppliers and catalogs.

This paper presents MCDM for evaluation mechanical ventilator devices by implementing TOPSIS and PROMETHEE II methods. TOPSIS \& PROMETHEE II are powerful techniques used to support decisions and handle evaluation some of alternative based of several criteria, with the aim of identifying the negatives and positives of alternatives and obtaining the order of alternatives and then obtaining the best alternative. 
TOPSIS \& PROMETHEE II are very easy to apply in engineering applications and in the medical decision. This methodology has proven to be the most appropriate and best way for decision-makers to evaluate and purchase mechanical ventilator by defining the important criteria/parameters for the device.

\section{$7 \quad$ References}

[1] Ware, L. B., and Matthay, M. A. (2000). The acute respiratory distress syndrome. N Engl J Med. 342: 1334-49.

[2] Amitali, A. (2020). Ventilator Management: Introduction to Ventilator Management, Modes of Mechanical Ventilation, Methods of Ventilatory Support". [cited 7 April 2020]. Available form: https://emedicine.medscape.com/article/810126-overview

[3] Rello, J., Ollendorf, D. A., Pster, F., Vera-Llonch, M., Bellm, L., Redman, R., and Kollef, M. H. (2002). Epidemiology and outcomes of ventilator associated pneumonia in a large US database. Chest, 122 (6): 2115-21. https://doi.org/10.1378/chest.122.6.2115

[4] WALSH, T. S., DODDS, S. \& MCARDLE, F. 2004. Evaluation of simple criteria to predict successful weaning from mechanical ventilation in intensive care patients. British Journal of Anesthesia, 92, 793-799. https://doi.org/10.1093/bja/aeh139

[5] Marini JJ, Crooke PS III, and Truwit JD. (1989). Determinants and limits of pressure-preset ventilation: a mathematical model of pressure control. J Appl Physiology. 67(3):1081-92. https://doi.org/10.1152/jappl.1989.67.3.1081

[6] Hwang, C. L., and Yoon, K. (1981). Methods for multiple attribute decision making. In Multiple attribute decision making pp: 58-191 Springer, Berlin, Heidelberg. https://doi. org/10.1007/978-3-642-48318-9_3

[7] Opricovic, S., and Tzeng, G.-H. (2004). Compromise solution by MCDM methods: A comparative analysis of VIKOR and TOPSIS. European Journal of Operational Research. 156(2): 445-455. https://doi.org/10.1016/s0377-2217(03)00020-1

[8] Liu, P., \& Wang, M. (2011). An extended VIKOR method for multiple attribute group decision making based on generalized interval-valued trapezoidal fuzzy numbers. Scientific Research and Essays. 6(4): 765-776. [cited 12 May 2020]. Available form: https://academicjournals.org/journal/SRE/article-full-text-pdf/974904023320.pdf

[9] J. Huang (2008). Combining entropy weight and TOPSIS method for information system selection. IEEE International Conference on Automation and Logistics pp: 1281-1284, September, Qingdao, China. https://doi.org/10.1109/ical.2008.4636483

[10] Medtronic Medical system. Puritan Bennett 980 ventilator brochure and technical specifications. Available from: https://www.medtronic.com/content/dam/covidien/library/us/en/product/acute-care-ventilation/puritan-bennett-980-ventilator-system-techspecifications.pdf. https://doi.org/10.1097/01.bmsas.0000483981.03551.04

[11] GE Medical system. Care scape R860 S1 ventilator brochure and technical specifications [cited 12 April 2020]. Available form: https://www.medtronic.com/covidien/en-us/products/mechanical-ventilation/puritan-bennett-980-ventilator.html

[12] Hamilton Medical system. G5/S1 ventilator brochure and technical specifications. Available from: https://www.hamilton-medical.com/dam/jcr:c9a80d84-fad0-46d0-9959-3b905048 c6c0/HAMILTON-G5-S1-quick-guide-en-627195.00.pdf

[13] Dräger Medical system. Evita V500 ventilator brochure and technical specifications. Available from: https://www.draeger.com/Products/Content/evita-infinity-v500-ventilator-pi9051753-en-gb.pdf. https://doi.org/10.1097/01.bmsas.0000450151.28761.6c

[14] Maquet Medical system. Servo-U ventilator brochure and technical specifications. Available from: https://www.getinge.com/siteassets/products-a-z/servo-u-mechanical-ventilator/ $\underline{\text { servo-u-datasheet-6886378_en-nonus-rev012.pdf?disclaimerAccepted=yes }}$ 
[15] J. P. Brans, B. Mareschal, and P. Vincke (1984). "PROMETHEE: a new family of outranking methods in MCDM, North Holland pp: 477-490.

[16] Macharis, C., Springael J., De Brucker, K., Verbeke, A., 2004: PROMETHEE and AHP: The design of operational synergies in multicriteria analysis. Strengthening PROMETHEE with ideas of AHP. European Journal of Operational Research 153: 307-317. https:// doi.org/10.1016/s0377-2217(03)00153-x

[17] C. Ting-Yu, "A PROMETHEE-based outranking method for multiple criteria decision analysis with interval type-2 fuzzy sets." Soft Computing, 18(5), 2014, pp. 923-940. https://doi.org/10.1007/s00500-013-1109-4

[18] H. Sonia and N. Halouani, "Hesitant-fuzzy-promethee method." In Modeling, Simulation and Applied Optimization (ICMSAO), 2013 5th International Conference on, pp. 1-6. IEEE, 2013. https://doi.org/10.1109/icmsao.2013.6552710

[19] Rogalewicz, V., \& Jurickova, I. (2014). Multiple-criteria decision making: application to medical devices. In IWBBIO (pp. 1359-1372).

[20] Karlsson, V., Bergbom, I., \& Forsberg, A. (2012). The lived experiences of adult intensive care patients who were conscious during mechanical ventilation: a phenomenological-hermeneutic study. Intensive and critical care nursing, 28(1), 6-15. https://doi.org/10.1016/ j.iccn.2011.11.002

[21] Terndrup, T. E., \& Rhee, J. (2006). Available ventilation monitoring methods during prehospital cardiopulmonary resuscitation. Resuscitation, 71(1), 10-18. https://doi.org/10.1016/ j.resuscitation.2006.02.019

\section{Authors}

Qasem Alyazji is currently a Ph.D. Student at Faculty of Engineering, Biomedical Engineer Department, Near East University, Nicosia / TRNC, Mersin 10-Turkey. He completed his B.Sc. degree in system \& biomedical engineering at Cairo University. Between the years 2008 and 2019 he worked as biomedical engineer as full time in ministry of health Palestine in Gaza Strip, Palestine. Between the years 2010 and 2012 he worked as lecturer in medical technician department in Nama'a College for Science \& Technology in Gaza, Palestine. Later on, he received his M.Sc. degree in biomedical engineer at Near East University. Between the years 2016 and 2019 worked as lecturer as part time in Biomedical Engineering Department in Gaza, Palestine. He worked as a lecturer in the Department of Medical Engineering Since the 2016 and 2020 at Alazhar University in Gaza, Palestine.

Gulsum Asiksoy, completed her undergraduate degree at the Ankara University Physics Engineering Department and her post graduate degree in the field of ElectricalElectronic Engineering. She obtained her doctorate degree (Computer Education and Instructional Technology, Near East Boulevard, 99138 Nicosia / TRNC) at the Near East University Computer Technologies Department. Assoc. Prof. Dr Asiksoy has published articles and books in the Sciences and Social Sciences indices in the fields of flipped learning, games in learning and teaching, interactive simulation in teaching physics, digital games-simulations.

Article submitted 2021-02-05. Resubmitted 2021-03-28. Final acceptance 2021-04-01. Final version published as submitted by the authors. 